\title{
Modeling Unavailability in Peer-to-Peer Systems
}

\author{
Daniel Sadoc Menasche*, Antonio A. de Aragao Rocha ${ }^{\dagger}$, Bin $\mathrm{Li}^{\ddagger}$, Don Towsley*, Arun Venkataramani* \\ ${ }^{*}$ University of Massachusetts, Amherst \{ sadoc, towsley, arun $\} @$ cs.umass.edu \\ $\dagger$ Federal University of Rio de Janeiro, Brazil arocha@land.ufrj.br \\ $\ddagger$ Tsinghua University, China libinhere@gmail.com
}

\begin{abstract}
In this paper we model peer-to-peer systems where content may become unavailable. First, using Markov Chains we capture the impact of parameters such as the server upload rate and the popularity of the files on the performance of the system. Then, we show the applicability of our model to the problem of optimal bundling. Bundling files together, rather than disseminating them separately, is a possible solution to improve availability in BitTorrent.
\end{abstract}

\section{INTRODUCTION}

Unstructured peer-to-peer systems have a number of properties which explain their tremendous popularity, e.g. the capacity of the system scales with the number of peers and peers can come and go in a random fashion. Nevertheless, when a peer leaves the system it may carry the last copy of a block of a file and for this reason it is hard to predict the availability of the files and consequently the performance of the system as a whole. In this article we model peer-topeer systems, à la BitTorrent, where content may become unavailable.

BitTorrent [1] uses P2P file swarming to disseminate content. A swarm is formed by a set of users interested in downloading a given file (or set of files, packaged in a bundle). The file is chopped into blocks that peers download from the server and from each other. A tracker is used to coordinate the interaction among the peers. Periodically, a peer queries the tracker and obtains a random subset of other peers in the swarm. A leecher is a peer that hasn't finished its download yet. After concluding the download, the leecher becomes a seed. Peers that have incentives to make the content available are referred to as publishers. The very first peer that makes available a given content is the first publisher.

The key contributions of this work are the following: (1) we propose a model to capture the effect of content unavailability on performance; (2) we show the applicability of our model to the problem of deciding the optimal bundling strategy for a publisher.

\section{One Publisher That Leaves And Never Returns}

We begin with the case of a single publisher that leaves and never returns. We introduce a simplifying assumption regarding the conditions for content availability, namely, that content becomes unavailable if and only if the number of peers in the system goes to zero (this assumption is eliminated in Section IV). Furthermore, for simplicity, we assume that the publisher and peers each remain in the system for an exponentially distributed period of time equal to the one of a typical peer. Under these conditions we can model this system as an $M / M / \infty$ queue. The period of time during which the content is available corresponds to the busy period of the queue. Let $\lambda$ be the arrival rate of peers and $1 / \mu$ the mean residence time. Then, the busy period is given by $\left(e^{\lambda / \mu}-1\right) / \lambda$.

\section{Intermittent Publishers With Impatient PeERS}

We now consider a system where publishers of the same content arrive according to a Poisson process with parameter $\gamma$. Each publisher remains for an average time $1 / \alpha$. Peers that arrive to the system when the content is not available leave immediately. We model the system as a Markov Chain with state $(l, p)$ where $l$ and $p$ stand for the number of leechers and publishers in the system, respectively. Starting from state $(l, p)$, the transitions are given as follows:

1) $l=0, p=0:(0,0) \stackrel{\gamma}{\rightarrow}(0,1)$

2) otherwise: $(l, p) \stackrel{\lambda}{\rightarrow}(l+1, p),(l, p) \stackrel{l \mu}{\rightarrow}(l-1, p)(l>0)$, $(l, p) \stackrel{\gamma}{\rightarrow}(l, p+1),(l, p) \stackrel{p \alpha}{\rightarrow}(l, p-1)(p>0)$

(Note that if $\lambda=\gamma$ and $\mu=\alpha$ we have an $M / M / \infty$ queue, as in the last section.) Using this model we can compute, for instance, the fraction of leechers that arrive to find the content unavailable, which is given by the probability of state $(0,0)$.

\section{One Intermittent Publisher with Patient LEECHERS}

In this section we consider the case where a single publisher for the content goes on and off. The state $(l, p, a)$ of the system is characterized by $l$, the number of leechers, $p$, the state of the publisher [on (1) or off (0)] and $a$, the content availability [available (1) or not (0)]. Starting from state $(l, p, a)$, the transitions go as follows:

1) $l>2$ :

$$
\begin{array}{ll}
\text { a) } & p=0, a=1:(l, 0,1) \stackrel{l \mu}{\rightarrow}(l-1,0,1),(l, 0,1) \stackrel{\lambda}{\rightarrow} \\
& (l+1,0,1),(l, 0,1) \stackrel{\gamma}{\rightarrow}(l, 1,1) \\
\text { b) } & p=0, a=0:(l, 0,0) \stackrel{\lambda}{\rightarrow}(l+1,0,0),(l, 0,0) \stackrel{\gamma}{\rightarrow} \\
& (l, 1,1) \\
\text { c) } & p=1, a=1:(l, 1,1) \stackrel{\lambda}{\rightarrow}(l+1,1,1),(l, 1,1) \stackrel{l \mu}{\rightarrow} \\
& (l-1,1,1),(l, 1,1) \stackrel{\alpha}{\rightarrow}(l, 0,1)
\end{array}
$$

2) $l=2$ :
a) $p=0, a=1:(2,0,1) \stackrel{2 \mu}{\rightarrow}(1,0,0),(2,0,1) \stackrel{\lambda}{\rightarrow}$ $(3,0,1),(2,0,1) \stackrel{\gamma}{\rightarrow}(2,1,1)$
b) otherwise: b) and c) for case $l>2$

3) $l=1$ : 

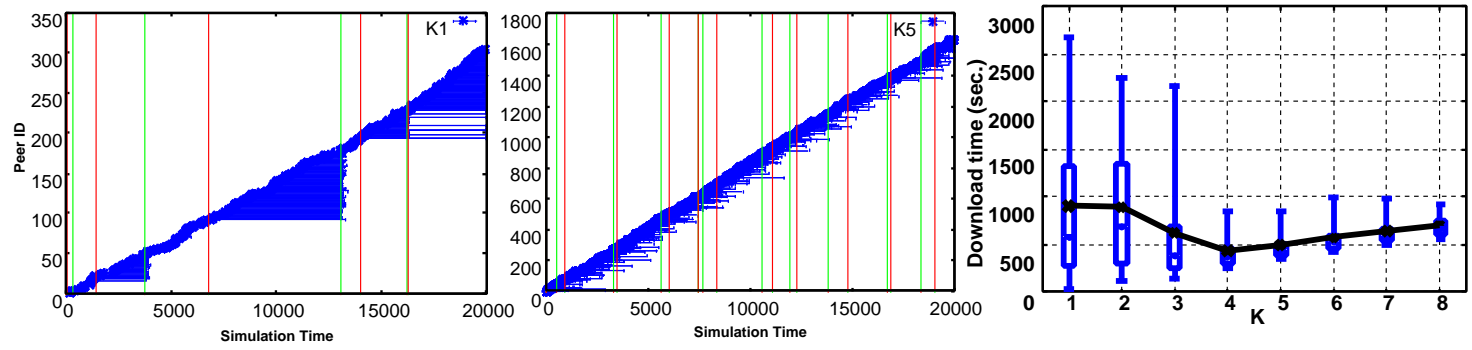

Fig. 1. (a) Simulation results for $\mathrm{K}=1$; (b) Simulation results for $\mathrm{K}=5$; (c) PlanetLab results.

a) $p=0, a=0:(1,0,0) \stackrel{\lambda}{\rightarrow}(2,0,0),(1,0,0) \stackrel{\gamma}{\rightarrow}$ $(1,1,1)$

b) $p=1, a=1:(1,1,1) \stackrel{\lambda}{\rightarrow}(2,1,1),(1,1,1) \stackrel{\alpha}{\rightarrow}$ $(1,0,0),(1,1,1) \stackrel{\mu}{\rightarrow}(0,1,1)$

4) $l=0$ :

a) $p=1, a=1:(0,1,1) \stackrel{\lambda}{\rightarrow}(1,1,1),(0,1,1) \stackrel{\alpha}{\rightarrow}$ $(0,0,0)$

b) $p=0, a=0:(0,0,0) \stackrel{\lambda}{\rightarrow}(1,0,0),(0,0,0) \stackrel{\gamma}{\rightarrow}$ $(0,1,1)$

Premature Content Unavailability: We now eliminate the assumption that content becomes unavailable only when the number of peers in the system goes to 0. For that purpose, we define a coverage threshold, $T$. We modify the Markov Chain presented in the last section to account for the fact that if the number of peers in the systems is less than $T$ there is a probability $q>0$ that content becomes unavailable.

- $T>l>2, p=1, a=1:(l, 1,1) \stackrel{\alpha q}{\rightarrow}(l, 0,1)$, $(l, 1,1) \stackrel{\alpha(1-q)}{\rightarrow}(l, 0,0)$

- $T>l>2, p=0, a=1:(l, 0,1) \stackrel{l \mu q}{\longrightarrow}(l-1,0,1)$, $(l, 0,1) \stackrel{l \mu(1-q)}{\rightarrow}(l-1,0,0)$

- other states: as in the previous model

\section{BUNDLING}

To show the applicability of our model, we consider the problem of deciding the optimal level of bundling in a peerto-peer system. Consider a server publishing $F$ files using swarming. The server has the flexibility to decide how to combine the files. Assume a homogeneous population of files each one with popularity $\lambda$ and size $s$. If $K$ files are bundled, the arrival rate to this swarm is $K \lambda$ and the size of the bundled file is $K s$.

We want to find the value of $K$ that minimizes the net download time. Note that small values of $K$ lead to an increase in download time without a significant improvement in availability, while large values of $K$ lead to large bundles, which necessarily will take longer to download.

We simulate the model presented in Section IV with $q=$ $1, T=11, \lambda=(1 / 60) K, \mu=(1 / 80) / K, \gamma=(1 / 300)$, $\alpha=(1 / 900)$ using Tangram II (http://www.land.ufrj.br). In Figures 1(a) and (b), each line segment starts at the instant the peer arrives and ends when the peer departs. Green and red vertical lines represent arrivals and departures of the publisher.
When $K=1$ (Figure 1(a)) many peers get blocked due to the unavailability of the content. When $K=5$ (Figure 1(b)), on the other hand, the swarm is self sustaining: even when the publisher is not available the peers still smoothly complete their downloads. For $K$ varying between 1 and 8, we obtained the following values for the expected download time of the peers: $(1586,1563,1336,522,412,487,568,650)$.

To evaluate the model against real experiments, we ran BitTorrent clients in PlanetLab under the same conditions as described in the previous paragraph. We varied the bundling factor $K$ from 1 up to 8. In Figure 1(c) we show the expected download time observed in the PlanetLab experiments as a function of $K$. The optimal bundling factor predicted by the model was close enough to the one observed in the experiments (4 and 5, respectively) and the linear increase in the download time for $K>5$ was correctly captured by the model.

\section{RELATED WORK}

[2] were the first to study the blocked leecher problem (BLP), i.e., the problem related to peers that have to wait for a long period of time for some blocks of a file that are not available. To address the problem they propose BitStore, a token based incentive architecture to obtain the missing blocks cached at other peers that had already downloaded the whole file. In this paper we propose bundling to mitigate the BLP.

[3]-[5] proposed models to analyze the performance of p2p systems using Markov Chains or differential equations. However, none of these models has taken content availability into account. [6] analyze file availability but their performance model does not take into account file unavailability due to churn.

\section{REFERENCES}

[1] B. Cohen, "Incentives build robustness in bittorrent," in Workshop on Economics of Peer-to-Peer Systems, 2003.

[2] A. Ramachandran, A. das Sarma, and N. Feamster, "Bitstore: An incentive compatible solution for blocked downloads in bittorrent," in Proc. Economics of Networked Systems and Incentive-Based Computing, 2007.

[3] X. Yang and G. Veciana, "Service capacity of peer to peer networks," in Infocom, 2004.

[4] Z. Ge, D. Figueiredo, S. Jaiswal, J. Kurose, and D. Towsley, "Modeling peer-peer file sharing systems," in Infocom, 2003.

[5] D. Qiu and R. Srikant, "Modeling and performance analysis of bittorrentlike peer-to-peer networks," in SIGCOMM, 2004.

[6] B. Fan, D.-M. Chiu, and J. Lui, "Stochastic analysis and file availability enhancement for bt like file sharing systems," in IWQoS, 2006. 